\title{
PLANIFICACIÓN DE TRAYECTORIAS PARA UAVS CON FAST MARCHING SQUARE ADAPTADAS A REQUERIMIENTOS DE VUELO
}

\author{
Verónica González, Concepción A. Monje, Luis Moreno y Carlos Balaguer \\ Universidad Carlos III, Leganés, Madrid, 28911, España, \\ \{vegonzal, cmonje, moreno, balaguer\}@ing.uc3m.es
}

\section{Resumen}

En este artículo se plantea un método destinado a la planificación de misiones globales para vehículos aéreos no tripulados (UAVs, por sus siglas en inglés) en función de unos requerimientos iniciales específicos. La planificación es realizada en un entorno 3D. La metodología seguida está basada en la implementación del algoritmo Fast Marching Square $\left(F M^{2}\right)$ para generar la ruta más corta en tiempo entre dos puntos, el cual incluye dos parámetros de ajuste. Estos dos parámetros de ajuste modificarán el primer potencial (W) generado por $F M^{2}$ para adaptar la trayectoria resultante de acuerdo a unas necesidades iniciales, como pueden ser, el ahorro de energía o combustible, o simplemente el rastreo de una zona específca, lo que da lugar a una minimización de costes en términos generales. Diferentes simulaciones se han llevado a cabo obteniendo buenos resultados.

Palabras clave: Fast Marching, Fast Marching Square, Planificación de misiones globales, Entorno 3D, Vehículo Aéreo no Tripulado.

\section{INTRODUCCIÓN}

En los últimos años, muchas investigaciones se han centrado en la utilización de los vehículos aéreos no tripulados (VANTs), o también conocidos por sus siglas en inglés como UAVs, en cantidad de aplicaciones. Esto es debido a que todas esas aplicaciones están destinadas a ayudar o a colaborar con el ser humano de una forma u otra. Entre ellas destacan tareas destinadas a la búsqueda y rescate de personas, acceso a lugares peligrosos o tareas de vigilancia. Incluso en el ambito más comercial, son utilizados para actividades de filmografía y fotografía.

Estas tareas normalmente tienen misiones concretas y la mayoría están destinadas a ser llevadas a cabo de forma autónoma. Para que éstas puedan ser ejecutadas, se requiere una adecuada planificación de trayectorias que cumpla con los objetivos de la misión. El ahorro de energía o combustible suele ser uno de los requisitos primordiales a la hora de planificar. Dicha planificación debe ser adaptada a la geometría del entorno, respentando siempre una determinada seguridad, es decir, evitando todo obstáculo encontrado en el camino y manteniendo distancias de seguridad respecto a los obstáculos. Sin embargo, en muchas ocasiones, el método utilizado para planificar no tiene en cuenta ciertos factores como son el ahorro de energía o combustible, o simplemente el rastreo de un zona específica.

Existen cantidad de estudios donde se utilizan diferentes técnicas para realizar planificación de trayectorias, sin embargo, las trayectorias generadas necesitan más iteraciones para ser mejoradas. Algunos de los algoritmos utilizados son Rapidly exploring Random Tree (RRT) [6], algoritmos de búsqueda $\mathrm{A}^{*}$ y $\mathrm{D}^{*}[10]$ o métodos probabilísticos de caminos (PRM) [7].

Este trabajo expone un método de planificación basado en Fast Marching Square $\left(\mathrm{FM}^{2}\right)$. Este algoritmo se ha utilizado en anteriores trabajos en planificación de trayectorias con robots móviles en entornos bidimensionales [3], [4] y tridimensionales [1], [5], obteniendo muy buenos resultados. Además también se ha demostrado que este algoritmo genera trayectorias suficientemente suaves y seguras para ser ejectuadas por un UAV [5]. A continuación se exponen las principales ventajas de este algoritmo:

- La trayectoria resultante es siempre la más óptima ya que el algoritmo genera una única onda donde solo existe un mínimo global.

- Las trayectorias generadas son muy suaves y seguras, lo que las hace totalmente ejecutables por un vehículo.

- Puesto que las trayectorias resultantes son muy suaves, no necesitan ser refinadas después, lo que asegura un bajo coste computacional.

El principal objetivo de esta investigación es la utilización del algoritmo $\mathrm{FM}^{2}$ en un entorno 3D para planificar misiones. Estas misiones tendrán un requisito primordial, como será la planificación 
de trayectorias por determinadas zonas del mapa, lo que puede ocasionar una trayetoria más óptima para un posible ahorro de energía.

Como punto de vista innovador, este método permite jugar con unos parámetros de ajuste para planificar las misiones por zonas específicas. Esto no solo supondrá la generación de trayectorias óptimas en términos de seguridad, si no que también serán optimizadas para minimizar los costes en la mision.

El artículo está organizado de la siguiente forma. La Sección 2 describe los métodos de planificación de trayectorias utilizados para la planificación de la misión. La Sección 3 explica la metodología a seguir para generar las trayectorias en función de unos requisitos específicos en un entorno 3D. En la Sección 4 se exponen los resultados obtenidos con éste método. Y por último, la Sección 5 presenta las conclusiones de este trabajo.

\section{MÉTODOS FAST MARCHING}

\subsection{MÉTODO FAST MARCHING}

El método Fast Marching (FMM) es un algoritmo numérico introducido por J.A Sethian [9] en 1996. Este método es un caso particular de Level set methods [8], el cual explica cómo se expande una onda en un determinado medio. Si consideramos un entorno bidimensional, el comportamiento de la onda está basado en la ecuación de la eikonal (1),

$$
1=W(x)|\nabla D(x)|
$$

donde $x$ es un punto determinado del espacio, $W(x)$ es la velocidad de expansión de la onda y $D(x)$ es el tiempo de llegada del frente de onda a cada punto del espacio. FMM calcula en cada una de sus iteraciones un tiempo $D_{i, j}$ para cada punto del espacio $x_{i, j}$ de acuerdo a la discretización del ecuación de la eikonal, donde $\Delta x$ y $\Delta y$ son el espaciado de las celdas de las direcciones de $x$ e $y$, respectivamente:

$$
\left(\frac{D-D_{1}}{\triangle x}\right)^{2}+\left(\frac{D-D_{2}}{\triangle y}\right)^{2}=\frac{1}{W_{i, j}^{2}},
$$

donde

$$
\begin{aligned}
& D=D_{i, j}, \\
& D_{1}=\min \left(D_{i-1, j}, D_{i+1, j}\right), \\
& D_{2}=\min \left(D_{i, j-1}, D_{i, j+1}\right) .
\end{aligned}
$$

Como resultado de este proceso se obtiene un mapa de distancias, también llamado Mapa de tiempos de llegada (Fig. 1a). Para obtener la trayectoria se emplea un descenso de gradiente, el cual

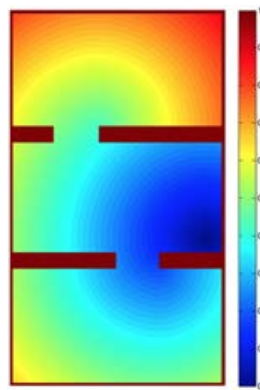

(a)

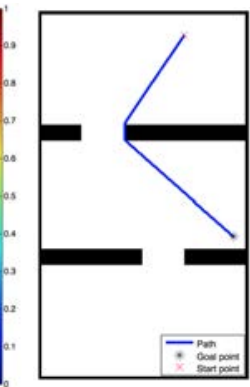

(b)
Figura 1: Aplicación del algoritmo FMM: (a) Mapa de distancias; (b) Trayectoria generada.

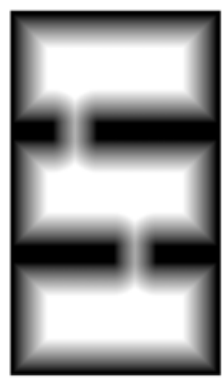

(a)

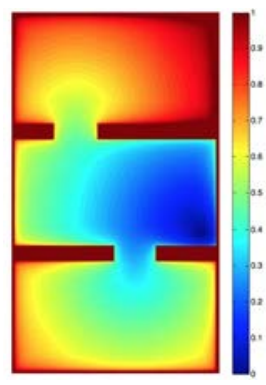

(b)

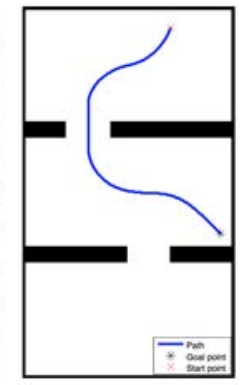

(c)
Figura 2: Aplicación del algoritmo $\mathrm{FM}^{2}$ : (a) Mapa de velocidades; (b) Mapa de tiempos de llegada; (c) Trayectoria generada.

genera una geodésica (Fig. 1b), es decir, la ruta más corta que existe entre dos puntos. El punto estipulado como fuente tendrá un valor de $D=0$, siendo éste el único mínimo global del entorno, y el resto de puntos serán $D>0$.

Sin embargo, la geodésica generada no disfruta de suavidad alguna, observándose cambios abruptos en las curvas. Tampoco proporciona distancias de seguridad a los obstáculos. Estos problemas ocasionados pueden ser resueltos por el algoritmo $\mathrm{FM}^{2}$, el cual es descrito a continuación.

\subsection{MÉTODO FAST MARCHING SQUARE}

El algoritmo $\mathrm{FM}^{2}$ fue introducido por Garrido et al. [2] en 2009, y se basa en aplicar dos veces el algoritmo FMM. A diferencia de FMM, este algoritmo genera trayectorias muy suaves y con una distancia de seguridad respecto de los obstáculos del entorno. A continuación se describe el proceso que sigue el algoritmo $\mathrm{FM}^{2}$ para generar una trayectoria:

1. El entorno 3D $W_{o}$ es representado como un mapa binario, donde las celdas pertenecientes a los obstáculos toman el valor 0 (negro) y el 
espacio libre toma el valor de 1 (blanco).

2. Se aplica el algoritmo FMM a cada una de las celdas de $W_{o}$ pertenecientes a los obstáculos. Como se resultado se obtiene un mapa de potenciales $W$, donde cada celda adquiere un valor entre 0 y 1 (Fig. 2a). Éste es también es denominado Mapa de velocidades, siendo el valor de cada celda proporcional a la distancia a los obstáculos.

3. FMM es aplicado de nuevo desde el punto final hasta que alcanza el punto inicial. Como resultado se obtiene un mapa de coste $D$, llamado también Mapa de tiempos de llegada (Fig. 2b).

4. Para obtener la trayectoria se aplica el descenso de gradiente sobre $D$ desde el punto inicial hasta el punto final (Fig. 2c). Esto da como resultado una aproximación muy buena de la geodésica, ya que proporciona la ruta más corta en función del $W$ generado.

Sin embargo, muchas veces la ruta generada no se adapta a nuestras necesidades, ya que tiene falta de naturalidad o no es ejecutable por un vehículo. Además, puede que la suavidad o los márgenes de seguridad a los obstáculos no sean adecuados. Para ello, en este trabajo proponemos la utilización de un parámetro de ajuste, el cual modifica $W$ adaptándolo a nuestras necesidades.

\subsubsection{Parámetro de ajuste}

El valor de cada celda de $W$ es elevado a un determinado valor al que llamamos parámetro de ajuste. Este parámetro modifica $W$ en función de nuestras necesidades, como puede ser la suavidad y la distancia a los obstáculos.

Si el parámetro de ajuste tiene un valor mayor que 1 , éste tenderá a oscurecer $W$, tal y como se aprecia al comparar las Figuras 3a y 3b. Esto provocará un mayor margen de seguridad con respecto a los obstáculos, pero sin embargo la trayectoria será menos suave. Por el contrario, si el parámetro tiene un valor menor que $1, W$ será aclarado, produciendo rutas más cercanas a los obstáculos con una mayor suavidad, como se muestra en la Fig. 3c.

\section{PLANIFICACIÓN EN FUNCIÓN DE REQUERIMIENTOS DE VUELO}

En esta sección se presenta la metodología seguida para planificar la misión en función de unos deter-

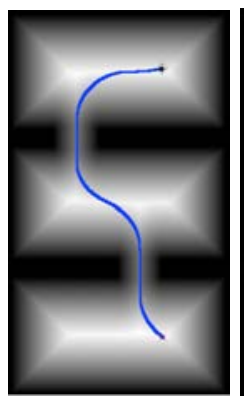

(a)

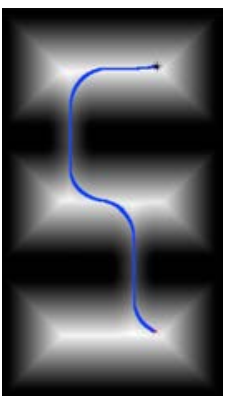

(b)

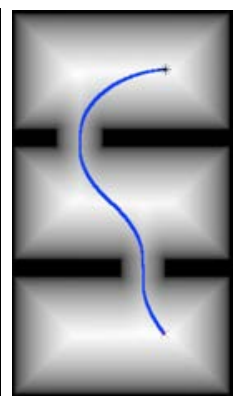

(c)
Figura 3: $W$ elevado a diferentes parámetros de ajuste: (a) $W$ elevado a 1 ; (b) $W$ elevado a 1.5 ; (c) $W$ elevado a 0.5 .

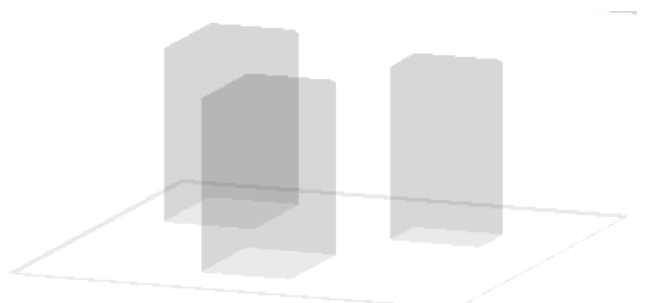

Figura 4: Entorno 3D donde se realizarán las simulaciones.

minados requerimientos. Las trayectorias son generadas en un entorno 3D mallado compuesto por tres obstáculos, el cual se muestra en la Fig. 4.

El algoritmo escogido como planificador es $\mathrm{FM}^{2}$, donde el uso del método del parámetro de ajuste es primordial para crear las trayectorias en función de unos requerimientos específicos. En nuestro caso, el requerimiento principal es que la planificación de la trayectoria se haga por zonas determinadas del mapa. Esto producirá un ahorro de energía en el UAV ya que evitará cambios de velocidad al mantenerse por una determinada capa del entorno. Para ello, se jugará con las distintas capas del mapa mallado. Si se desea planificar la trayectoria por una zona en concreto, la capa perteneciente a esta zona debe ser aclarada, ya que el frente de onda del algoritmo tiende a ir por las zonas más claras. El resto del área debe ser oscurecida, dificultando así la expansión de la onda.

Una vez que nuestro entorno es leído como un mapa binario, se aplica FMM sobre éste, generando $W$, como se ha explicado en la Sección 2.2. En este momento, $W$ es modificando en función de nuestros requerimientos. Para planificar la trayectoria por una capa en concreto, se utilizan dos parámetros de ajuste, donde $\rho_{1}$ es el encargado de aclarar y $\rho_{2}$ es el cargado de oscurecer, siendo siempre $\rho_{1}$ $<\rho_{2}$. Cada una de las celdas pertenecientes a $W$ serán elevadas a $\rho_{1}$ o a $\rho_{2}$, depediendo de la capa 


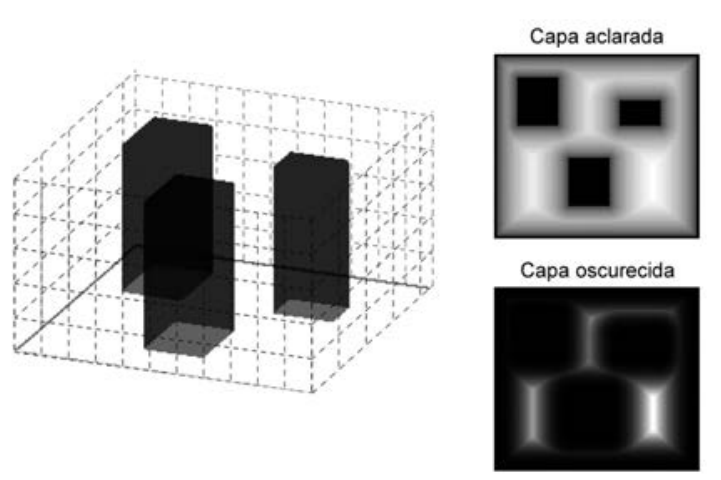

Figura 5: Ejemplo de aclarado y ocurecimiento de distintas capas del eje $z$.

a la que pertenezcan.

El Algoritmo 1 muestra el proceso para obtener el aclaramiento y oscurecimiento de las diferentes capas, como se muestra en la Fig. 5. Primero se define el número de capa $(\eta)$ en $W$ que se desea aclarar, así como la dirección de la capa $(\alpha)$, pudiendo pertenecer al eje $x, y$ o $z$. Cada una de las celdas $(w)$ de la capa escogida de $W$ son aclaradas elevando el valor de la celda a $\rho_{1}$, mientras que el resto de celdas son oscurecidas con $\rho_{2}$.

Cabe destacar que la diferencia existente entre los valores dados a $\rho_{1}$ y $\rho_{2}$ también afecta a la suavidad de la trayectoria. Si ambos valores distan mucho el uno del otro, la trayectoria tendrá curvas muy abruptas. Por el contrario, si $\rho_{1}$ y $\rho_{2}$ tienen valores cercanos, entonces la trayectoria será más suave.

Una vez que se ha aclarado toda la capa escogida, se aplica FMM a $W$ generando entonces la trayectoria en función de como ha sido modificado $W$.

\section{RESULTADOS DE LAS SIMULACIONES}

En esta sección se presentan los resultados obtenidos del método descrito en este trabajo, realizando una simulación de manera offline. El entorno donde se realizará la simulación ha sido descrito en la Sección 3.

Dos experimentos diferentes se han llevado a cabo. El primer experimento planifica la misión por zonas determinadas del entorno mallado. El segundo experimento muestra cómo en función de los valores dados a los parámetros de ajuste la suavidad y márgenes de seguridad de la trayectoria se ven afectados.
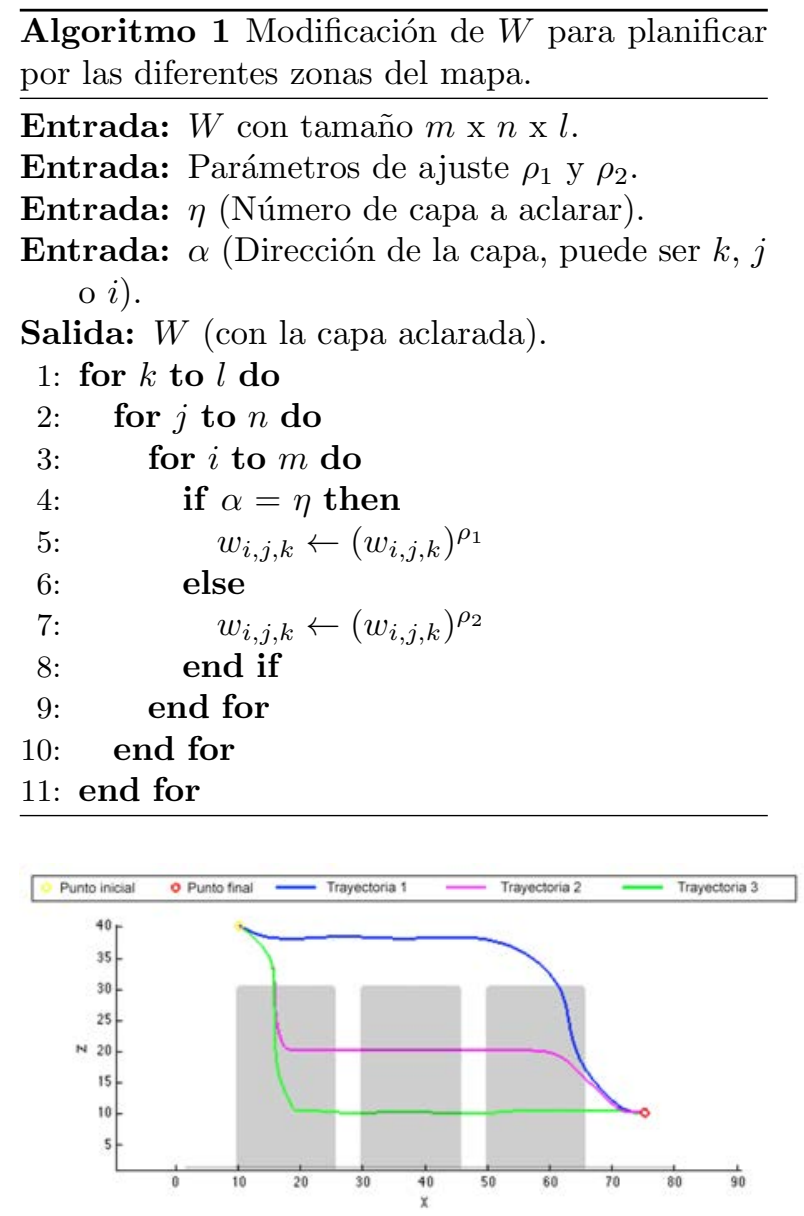

Figura 6: Diferentes planificaciones en distintas capas del eje $z$.

\subsection{PLANIFICACIÓN EN DIFERENTES CAPAS}

El principal objetivo de este experimento es planificar una trayectoria óptima entre los obstáculos por unas zonas específicas. Para ello, se planificará por distintas capas de los diferentes ejes de coordenadas, $x, y, z$.

\subsubsection{Planificación en capas del eje $\mathrm{z}$}

En este primer caso, se realizarán diferentes planificaciones en las distintas capas del eje $z$. Para ello, se han elegido dos capas diferentes, 10 y 20. Si se observa la Fig. 6 se pueden ver las diferentes trayectorias realizadas por diferentes zonas.

La Trayectoria 1 es representada por el color azul. En este caso, $W$ no ha sido modificado, por lo que la trayectoria generada sería la trayectoria natural del algoritmo $\mathrm{FM}^{2}$.

En el caso de la Trayectoria 2, representada por el color verde, $W$ ha sido modificado, aclarando la capa 10 con un valor de $\rho_{1}=0.3$, mientras que el resto de las capas han sido oscurecidas con un 


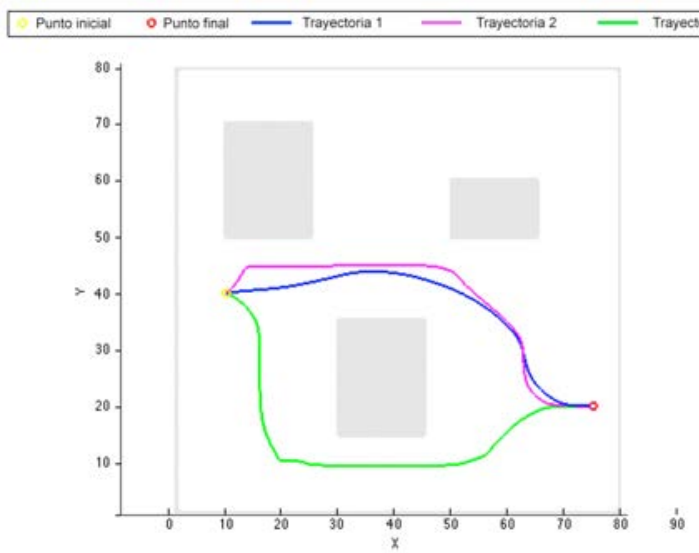

Figura 7: Diferentes planificaciones en distintas capas del eje $y$.
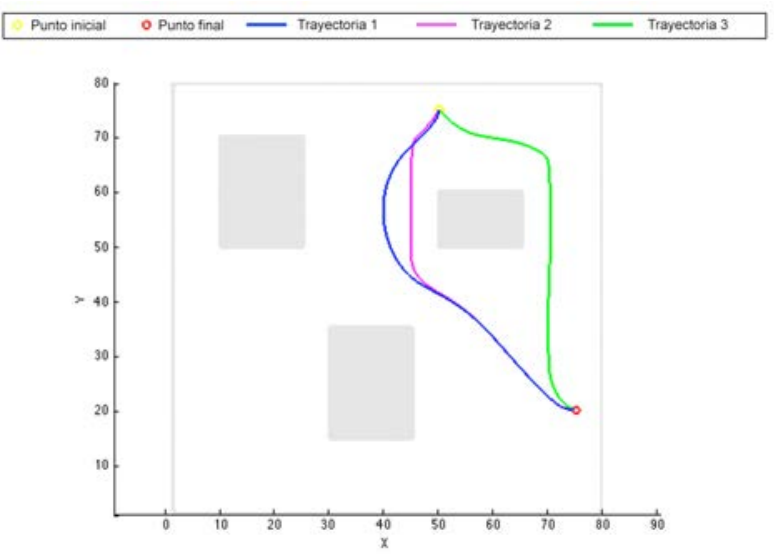

Figura 8: Diferentes planificaciones en distintas capas del eje $x$.

valor de $\rho_{2}=2$. Se puede apreciar cómo la trayectoria es mantenida por la capa número 20.

La Trayectoria 3 es representada en color magenta. En este caso, $W$ ha sido modificado aclarando la capa 10 con los mismos valores de $\rho_{1}$ y $\rho_{2}$ que en el caso de la Trayectoria 2. También es apreciable que la trayectoria es mantenida por esa capa.

\subsubsection{Planificación en capas del eje y}

El segundo caso es mostrado en la Fig. 7, donde las diferentes planificaciones son realizadas en distintas capas del eje $y$. En este caso, se han escogido las capas 45 y 10.

Al igual que el primer caso, la Trayectoria 1, representada en azul, mantiene la planificaión natural de $\mathrm{FM}^{2}$ ya que $W$ no ha sido modificado.

La Trayectoria 2, representada en color verde, es planificada por la capa 45 . Esta capa de $W$ ha sido aclarada con $\rho_{1}=0.3$ y el resto de capas oscurecidas con $\rho_{2}=2$.
Por último, se observa que la Trayectoria 3, en color magenta, es planificada por la capa 10 . En este caso, se ha aclarado esa capa de $W$ con los mismos valores de $\rho_{1}$ y $\rho_{2}$ que en la Trayectoria 2 .

\subsubsection{Planificación en capas del eje $\mathrm{x}$}

En este último caso se van a planificar las trayectorias por las diferentes capas del eje $x$, siendo éstas las capas 45 y 70 . Este caso es mostrado en la Fig. 8.

Al igual que en los casos anteriores, la Trayectoria 1 (azul) es planficada de forma natural, sin modificar $W$.

Para generar la Trayectoria 2 (magenta), se ha aclarado la capa 45 de $W$, manteniendo así la planificación por dicha capa. Los valores dados a $\rho_{1}$ y $\rho_{2}$ son 0.1 y 0.6 , respectivamente.

Y por último, para la Trayectoria 3 (verde) se ha aclarado la capa 70 de $W$, dando los mismos valores a $\rho_{1}$ y $\rho_{2}$ que en el caso de la Trayectoria 2 .

\subsection{CAMBIOS EN LA SUAVIDAD DE LA TRAYECTORIA}

En este segundo experimento se demuestra cómo la trayectoria puede ser suavizada en función de los valores dados a los parámeteros de ajuste. De esta forma, se podrá controlar la seguridad de la trayectoria para asegurar que sea ejectuable, por ejemplo, por un UAV.

En la Fig. 9 se puede apreciar la existencia de nuevo de tres trayectorias. Las tres están planificadas por una determinada capa. El valor de $\rho_{2}$ en todas las trayectorias se ha mantenido a 2.5, mientras que $\rho_{1}$ ha oscilado. En la Trayectoria 1 (azul) se ha impuesto $\rho_{1}=1$, en la Trayectoria 2 (magenta) $\rho_{1}=0.6$ y en la Trayectoria 3 (verde) $\rho_{1}=0.3$.

En la Fig. 9a se puede apreciar que no todas tienen la misma inclinación en el primer descenso. En este caso, la trayectoria con mayor inclinación de descenso es Trayectoria 3 , cuyos valores de $\rho_{1}$ y $\rho_{2}$ son los que más distan. Le sigue la Trayectoria 2 con una menor inclinación en el descenso. Los valores de $\rho_{1}$ y $\rho_{2}$ distan menos que en la Trayectoria 1. Y por último, si se observa la Trayectoria 1, es la que menos inclinación de descenso tiene, debido a que los valores de $\rho_{1}$ y $\rho_{2}$ son los que menos distan entre sí.

Si ahora se observa la Fig. 9b, las trayectorias no tienen la misma suavidad, y tampoco tienen la misma distancia respecto de los obstáculos. Si comparamos el valor de $\rho_{1}$ en las tres trayecto- 


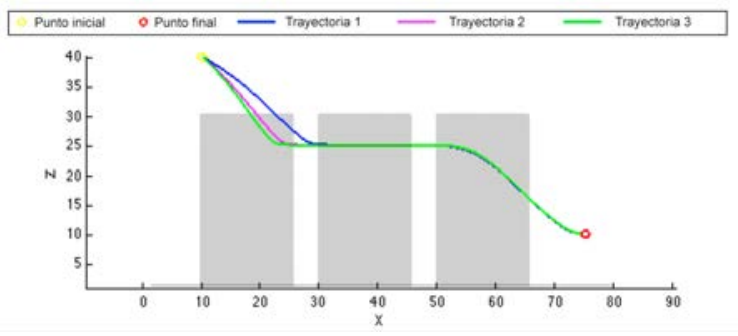

(a)

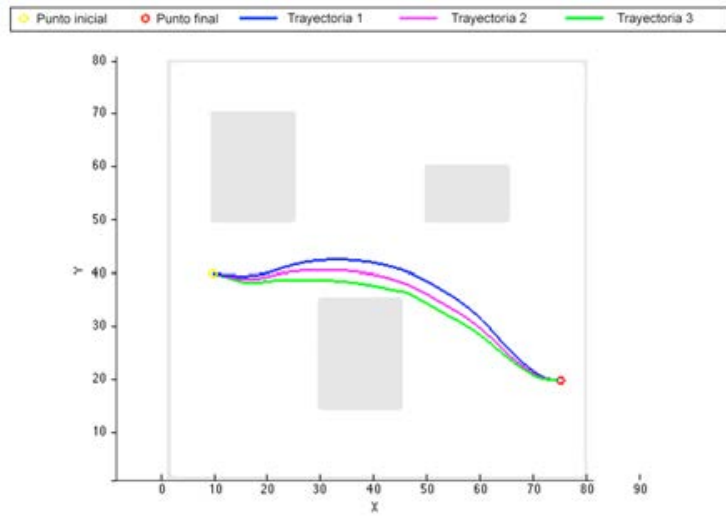

(b)

Figura 9: Variación de la trayectoria en función de los parámeteros de ajuste.

rias, se aprecia que cuanto menor es el valor de $\rho_{1}$, mayor es la aclaración en la capa y por lo tanto, mayor es la cercanía de la trayectoria a los obstáculos.

\section{CONCLUSIONES}

Esta investigación presenta un método novedoso basado en el algoritmo $\mathrm{FM}^{2}$ para modificar la trayectoria generada en un entorno 3D en función de unas necesidades específicas. El método planifica la trayectoria en función de unos parámetros de ajuste $\rho_{1}$ y $\rho_{2}$ utilizados para forzar a que la planificación se realice por una zona determinada. Esta variación de $\rho_{1}$ y $\rho_{2}$ causa modificaciones en $W$, lo que produce un oscurecimiento o aclaramiento de capas especificadas. Si se desea planificar por una zona específica, la capa de esa zona será aclarada, mientras que el resto de capas serán oscurecidas. De esta forma, $\mathrm{FM}^{2}$ siempre tenderá a planificar por las zonas más claras. Se ha comprobado igualmente que la suavidad de la trayectoria también puede ser modificada por estos parámetros de ajuste, produciendo rutas más seguras.

\section{Agradecimientos}

Este trabajo ha sido financiado por el proyecto RoboCity2030-III-CM (Robótica aplicada a la mejora de la calidad de vida de los ciudadanos. Fase
III; S2013/MIT-2748), financiado por el Programa de Actividades I+D Madrid y cofinanciado por los Fondos Estructurales de la Unión Europea.

\section{Referencias}

[1] Álvarez, D., Gómez, J.V., Garrido, S. y Moreno, L., (2014) "3D Robot Formations Planning with Fast Marching Square," Proceedings of the IEEE International Conference on Autonomous Robot Syst. and Competitions, Espinho, Portugal, pp. 59-64.

[2] Garrido,S., Moreno, L., Abderrahim, M., y Blanco, D., (2009) "FM2: a real-time sensorbased feedback controller for mobile robots," International Journal of Robotics and Automation, Vol. 24, No. 1, pp. 48-65. doi: 10.2316/Journal.206.2009.1.206-3169

[3] Garrido,S., Malfaz, M., y Blanco, D., (2013) "Application of the fast marching method for outdoor motion planning in robotics," Journal of Robotics and Autonomous Systems, Vol. 61, No. 2, pp. 106-114. doi:10.1016/j.robot.2012.10.012

[4] Gómez, J.V., Lumbier, A., Garrido, S., y Moreno, L., (2013) "Planning Robot Formations with Fast Marching Square including Uncertainty Conditions," Journal of Robotics and Autonomous Systems, Vol. 61, No. 2, pp. 137152. doi:10.1016/j.robot.2012.10.009

[5] González, V., Monje, C. A., Moreno, L., y Balaguer, C., (In press) "Fast Marching Square Method for UAVs Mission Planning with consideration of Dubins Model Constraints," Proceedings of the 20th IFAC Symposium on Automatic Control in Aerospace, Sherbrooke, Canadá.

[6] Lin, Y., and Saripalli, S., (2014) "Path planning using 3D dubins curve for unmanned aerial vehicles," en Int. Conf. on Unmanned Aircraft Systems, 2014, pp. 296-304.

[7] Obermeyer, K., Oberlin, P., and Darbha, S., (2012) "Sampling-based path planning for a visual reconnaissance unmanned air vehicle," Journal of Guidance, Control, and Dynamics, Vol. 35, No. 2, pp. 619-631.

[8] Osher, S., y Sethian, J.A., (1988) "Fronts propagating with curvature-dependent speed-algorithms based on Hamilton-Jacobi formulations," Journal of Computational Physics, Vol. 79, No. 1, pp. 12-49. 
[9] Sethian, J.A., (1996) "A fast marching level set method for monotonically advancing fronts," Proceedings of the Natural Academy of Science (USA), Vol. 93, No. 4, pp. 1591-1595.

[10] Xue, Q., Cheng, P., and Cheng, N., (2014) "Offline path planning and online replanning of uavs in complex terrain*," Proceedings of the 2014 IEEE Chinese Guidance, Navigation and Control Conference, pp. 2287 - 2292. 